- Original Article

\title{
Milk Consumption and Bone Mineral Density in Adults: Using Data from the Korea National Health and Nutrition Examination Survey 2008-2011
}

\author{
Ji Soo Kim', Seung-Won Oh',*, Jiwoo Kim ${ }^{3}$ \\ 'Department of Family Medicine, Seoul National University Hospital, Seoul, Korea \\ ${ }^{2}$ Department of Family Medicine, Healthcare System Gangnam Center, Seoul National University Hospital, Seoul, Korea \\ ${ }^{3}$ Seoul Barun Clinic, Seoul, Korea
}

Background: Milk consumption is associated with bone mineral density (BMD), but reports are limited in terms of participant age, sex, and number of study subjects. We investigated the association between milk consumption and BMD in South Korean adults ( $\geq 20$ years).

Methods: We analyzed men and women aged $\geq 20$ years who participated in the Korean National Health and Nutrition Examination Survey, 2008-2011. We used linear regression to calculate the mean BMD and 95\% confidence interval (CI) based on the frequency of milk consumption. Multivariate logistic regression analysis was used to estimate the odds ratios (ORs) and $95 \% \mathrm{CI}$ for T-scores $\leq-2.5$ (osteoporosis) in both men aged $\geq 50$ years and postmenopausal women.

Results: In total 8,539 subjects were studied. Drinking milk more than once a day was associated with higher BMD in the total femur and femoral neck in men aged $<50$ years and lumbar spine in men aged $\geq 50$ years, compared to less than once a week. It was also associated with lower ORs for osteoporosis of the femoral neck and lumbar spine in men aged $\geq 50$ years (OR, 0.35 ; $95 \% \mathrm{CI}, 0.125-0.979$ and OR, $0.34 ; 95 \% \mathrm{CI}, 0.143-0.804$, respectively). In postmenopausal women who consumed milk 2-6 times weekly, higher BMD and lower OR for osteoporosis were observed in the total femur (OR, 0.23; 95\% CI, 0.055-0.958).

Conclusion: This study suggests that frequent milk consumption could potentially reduce osteoporosis incidence in South Korean adults. Further prospective study is necessary to elucidate the effect of milk consumption on BMD.

Keywords: Milk; Bone Density; Osteoporosis; Adults

Received: July 28, 2020, Revised: November 19, 2020, Accepted: December 3, 2020

${ }^{*}$ Corresponding Author: Seung-Won Oh https://orcid.org/0000-0003-3800-0754

Tel: +82-2-2112-5643, Fax: +82-2-2112-5794, E-mail: sw.oh@snu.ac.kr 


\section{INTRODUCTION}

In metabolic bone diseases such as osteopenia and osteoporosis, bone mineral density (BMD) decreases, while the susceptibility to fractures increases. Osteoporosis and other related bone fractures are recognized as a global disease burden; in South Korea, the prevalence of osteoporosis was reported to be $7.5 \%$ and $35.5 \%$ in men and women, respectively. ${ }^{1)}$ While genetic predispositions for low BMD and osteoporosis have been previously reported, modifiable factors such as lifestyle modification in nutritional intake can mitigate these tendencies.

Dietary calcium has been reported to prevent osteoporosis and osteoporotic fractures. ${ }^{2-4)}$ According to the 2015 Dietary Reference Intakes for Koreans, proposed by the Korean Nutrition Society, a daily calcium intake of 700-800 mg or above is recommended for adults. ${ }^{5)}$ Milk is a major source of calcium. One cup of milk (approximately 200 $\mathrm{g}$ of milk) contains approximately $210 \mathrm{mg}$ of calcium, which is onethird of the recommended daily intake of calcium. Milk consumption in South Korea has increased since 1960; however, dairy products are not part of the traditional Korean diet, and milk consumption per capita has steadily declined from 1997 to $2015 .{ }^{6)}$ According to the Korea National Health and Nutrition Examination Survey (KNHANES) 2018, the daily calcium intake in Korean men and women (572 mg and 459 $\mathrm{mg}$, respectively) was inadequate compared to the aforementioned recommended intake. ${ }^{7)}$

Cross-sectional studies in Caucasian women aged 18-31 years ${ }^{8)}$ and 20-49 years $^{9)}$ reported that milk consumption during childhood and adolescence was related to greater bone mass. In a study of Chinese adolescent girls aged 12-14 years, milk intake was shown to be a better nutritional determinant of bone mineral content than the intake of any particular nutrient found in milk. ${ }^{10)}$ The association between milk and $\mathrm{BMD}$ has also been studied in the Korean population. Studies on female college students, ${ }^{11)}$ perimenopausal women (aged $40-70$ years), and postmenopausal women aged 50 years or above indicated that milk promoted optimal BMD. ${ }^{12)}$ However, these studies assessed only women of particular age groups, and the study populations used were mostly small. This cross-sectional study aimed to determine whether the frequency of milk consumption is associated with BMD in the general adult population (aged 20 years or above) in South Korea.

\section{METHODS}

\section{Research Plan and Study Population}

The KNHANES is a national cross-sectional survey conducted by the Korea Centers for Disease Control and Prevention. The process of selecting household units and participants was based on a stratified, multi-stage, clustered probability sampling design considered to be representative of the non-institutionalized population of South Korea. The data used in this study were obtained from the 2008 to 2011 KNHANES, in which BMD was assessed using dual-energy X-ray absorptiometry (DXA). The study population included both male and female adults aged 20 years or older. Participants diagnosed with thyroid disease, osteoporosis, rheumatoid arthritis, chronic liver disease, chronic kidney disease, or cancer were excluded, as were those who did not provide the necessary data during the survey. Finally, the study population comprised 8,849 men and women (Figure 1).

\section{Measurements of Anthropometric Parameters}

The anthropometric measurements used in the KNHANES were made by well-trained nurses and, in addition to the age of the participants, included the following: height (SECA 225; Vogel \& Halke, Hamburg, Germany), weight (GL-6000-20; CAS Korea, Seoul, Korea), and body mass index (BMI) (weight/height $\left.{ }^{2}\left[\mathrm{~kg} / \mathrm{m}^{2}\right]\right)$.

\section{Consumption of Milk and Calcium}

The frequency of milk consumption, including milk-based recipes, was categorized using one of the following categories: never, 6-11 times per year, once per month, 2-3 times per month, once per week, 2-3 times per week, 4-6 times per week, once per day, twice per day, 3 times per day, or unsure/no response. After excluding data from the unsure/no response category, participants who consumed milk were further divided into three groups: once per week or less, 2-6 times per week, and more than once per day. A 24-hour diet recall method and nutrition database that includes packaged food were used to quantify overall dietary and calcium intake.

\section{Bone Mineral Density}

The Hologic DXA System (Discovery QDR 4500W; Hologic Inc., Denver, Co, USA) was used to measure the BMD of the lumbar spine (L1L4), the neck of the femur, and the total femur. The values obtained

$\longrightarrow$\begin{tabular}{|l|l|}
$\begin{array}{l}\text { Excluded }(n=19,523) \\
\text { - Missing values for DXA }(n=9,892) \\
\text { - Diagnosed with thyroid disease, osteoporosis, rheumatoid arthritis, } \\
\text { chronic liver disease, chronic kidney disease, or cancer }(n=2,200) \\
\text { - Missing values for frequency of milk consumption }(n=2,297) \\
\text { - Missing values for covariates }(n=5,143)\end{array}$ \\
\hline
\end{tabular}

Study population $(n=8,539)$
Figure 1. Flow diagram of the study subjects. DXA, dual-energy X-ray absorptiometry. 
were recorded at five decimal places.

\section{Other Variables Related to Bone Mineral Density}

Potential confounding variables such as alcohol intake were categorized as one of the following: no intake within 1 year, less than once a month, once per month, 2-4 times per month, 2-3 times per week, and 4 times or more per week. Smoking status, menopause, and hormone treatment were also recorded in the survey. Serum levels of vitamin D were measured using a gamma counter (1470 Wizard; PerkinElmer, Turku, Finland) using a radioimmunoassay kit. Data pertaining to physical activity during the previous week (number of days and hours of walking, vigorous-intensity exercise, or moderate-intensity exercise) were collected and converted into metabolic equivalents of task-minutes per week. Diabetes was defined using the questionnaire as a case, which was diagnosed by doctors in the past.

\section{Statistical Analysis}

Statistical analyses were conducted using STATA/SE ver. 16.0 (Stata Corp., College Station, TX, USA). Weighted variables were used with regard to the composite stratified samples. We used linear regression to calculate mean BMDs and 95\% confidence intervals (CIs) regarding the frequency of milk consumption. We also considered the following potential confounding covariates: age, sex, BMI, physical activity, serum vitamin $\mathrm{D}$, calcium intake, smoking, alcohol intake, and diabetes. Multivariate logistic regression analyses were conducted to estimate the odds ratios (ORs) and 95\% CIs for T-scores of -2.5 or less (osteoporosis) in men aged 50 years or above and post-menopausal women with regard to the frequency of milk consumption. The lowest intake category was used as a reference.

\section{Ethical Considerations}

The Seoul National University Hospital Institutional Review Board approved this study (IRB approval no., E-2007-070-1141). The requirement for informed consent from the participants was waived, as the KNHANES database is anonymized according to strict confidentiality guidelines.

\section{RESULTS}

Table 1 lists the baseline characteristics of the study population. The

Table 1. Baseline characteristics of the study population

\begin{tabular}{|c|c|c|c|c|c|}
\hline \multirow{2}{*}{ Characteristic } & \multirow{2}{*}{ Total } & \multicolumn{3}{|c|}{ Frequency of milk consumption } & \multirow{2}{*}{ P-value } \\
\hline & & $\leq 1 /$ wk & $2-6 / w k$ & $\geq 1 / d$ & \\
\hline \multicolumn{6}{|l|}{ Men } \\
\hline No. of study population & 5,684 & 3,505 & 1,274 & 905 & \\
\hline Age $(y)$ & $49.5 \pm 15.9$ & $52.6 \pm 15.4$ & $45.0 \pm 15.3$ & $44.2 \pm 15.5$ & 0.890 \\
\hline Body mass index $\left(\mathrm{kg} / \mathrm{m}^{2}\right)$ & $24.0 \pm 3.1$ & $23.9 \pm 3.1$ & $24.2 \pm 3.2$ & $24.0 \pm 3.1$ & 0.414 \\
\hline Plasma vitamin D (ng/mL) & $20.3 \pm 7.1$ & $20.5 \pm 7.3$ & $19.9 \pm 7.1$ & $20.0 \pm 6.8$ & 0.054 \\
\hline Calcium intake $(\mathrm{mg} / \mathrm{d})$ & $569.1 \pm 363.5$ & $524.7 \pm 349.9$ & $602.1 \pm 357.8$ & $694.8 \pm 388.7$ & $<0.001$ \\
\hline Physical activity (MET min/wk)* & $3,644.2 \pm 5,251.0$ & $3,610.1 \pm 5,307.8$ & $3,722.8 \pm 5,161.8$ & $3,665.5 \pm 5,158.3$ & 0.346 \\
\hline Alcohol drinking frequency (\%) & & & & & $<0.001$ \\
\hline Never or none within 1 year & 871 & $590(67.7)$ & $159(18.3)$ & $122(14.0)$ & \\
\hline 4 or less than 4 times per month & 2,619 & $1,443(55.1)$ & $668(25.5)$ & $508(19.4)$ & \\
\hline 2 or more times per week & 2,194 & $1,472(67.1)$ & $447(20.4)$ & $275(12.5)$ & \\
\hline Current smoking (\%) & 3,644 & $2,282(62.6)$ & $807(22.1)$ & $555(15.2)$ & 0.087 \\
\hline Diabetes (\%) & 472 & 301 (63.8) & $100(21.2)$ & $71(15.0)$ & 0.282 \\
\hline \multicolumn{6}{|l|}{ Women } \\
\hline No. of study population & 2,855 & 1,453 & 797 & 605 & \\
\hline Age (y) & $46.6 \pm 14.7$ & $49.7 \pm 15.0$ & $43.7 \pm 13.8$ & $42.7 \pm 13.5$ & $<0.05$ \\
\hline Body mass index $\left(\mathrm{kg} / \mathrm{m}^{2}\right)$ & $23.3 \pm 3.5$ & $23.5 \pm 3.5$ & $23.2 \pm 3.5$ & $22.9 \pm 3.3$ & 0.111 \\
\hline Plasma vitamin D (ng/mL) & $16.1 \pm 6.0$ & $16.0 \pm 6.1$ & $16.1 \pm 5.9$ & $16.3 \pm 5.6$ & $<0.05$ \\
\hline Calcium intake (mg/d) & $475.1 \pm 311.3$ & $404.7 \pm 273.5$ & $495.5 \pm 315.2$ & $617.1 \pm 338.1$ & $<0.001$ \\
\hline Physical activity (MET min/wk)* & $2,187.0 \pm 3,498.6$ & $2,187.8 \pm 3,650.7$ & $2,194.6 \pm 3,554.3$ & $2,175.0 \pm 3,024.8$ & $<0.001$ \\
\hline Alcohol drinking frequency (\%) & & & & & $<0.05$ \\
\hline Never or none within 1 year & 905 & $492(54.4)$ & $227(25.1)$ & $186(20.6)$ & \\
\hline 4 or less than 4 times per month & 1,691 & $821(48.6)$ & $504(29.8)$ & $366(21.6)$ & \\
\hline 2 or more times per week & 259 & $140(54.1)$ & $66(25.5)$ & $53(20.5)$ & \\
\hline Current smoking (\%) & 152 & 77 (50.7) & $37(24.3)$ & $38(25.0)$ & 0.399 \\
\hline Diabetes (\%) & 147 & 93 (63.3) & $29(19.7)$ & $25(17.0)$ & $<0.05$ \\
\hline Menopause $(\%)^{\dagger}$ & 1,164 & $712(61.2)$ & $279(24.0)$ & $173(14.9)$ & $<0.001$ \\
\hline Hormone therapy (\%) & 230 & $117(50.9)$ & $71(30.9)$ & $42(18.3)$ & 0.408 \\
\hline
\end{tabular}

Values are presented as mean \pm standard deviation or number (\%), unless otherwise stated. P-values were derived by analysis of variance and Pearson's chi square test. MET, metabolic equivalent of task.

*Physical activity was used, according to the protocol of the Korean version of the International Physical Activity Questionnaire-Short Form, to calculate MET-minutes per week. †In menopause, surgical menopause was included. 
mean age of the male and female study population was 49.5 and 46.6 years, respectively. The mean calcium intake was lowest in the group who drank milk once per week or less in both the male and female study population. Women who drank milk more than once a day were younger and had lower physical activity than other groups, but this difference did not appear in men. Tables 2 and 3 shows the adjusted mean BMD values for the total femur, femoral neck, and lumbar spine according to the frequency of milk consumption. High levels of milk consumption, defined as more than once per day, were associated with higher BMD of the total femur and femoral neck in men aged $<50$ years. Milk consumption was also significantly associated with a higher BMD of the lumbar spine in men aged 50 years or above and in the total femur in postmenopausal women. In premenopausal women, no significant association was observed between BMD and the frequency of milk consumption.

The ORs for osteoporosis (T-score <-2.5) according to the frequency of milk consumption in both men aged 50 years or above and postmenopausal women are shown in Table 4 . In both groups, milk consumption was associated with a reduced OR for osteoporosis. In men, the ORs for osteoporosis of the femoral neck and lumbar spine were significantly lower in the group with the highest intake (OR, 0.35; 95\% CI, 0.125-0.979 and OR, 0.34; 95\% CI, 0.143-0.804, respectively). In women, the OR for osteoporosis of the total femur was significantly lower in the group that consumed milk 2 to 6 times per week than in the lowest intake group (OR, 0.23; 95\% CI, 0.055-0.958).

\section{DISCUSSION}

Our study examined the association between the frequency of milk consumption and BMD in Korean adults, including both young adults acquiring peak bone mass and older adults undergoing bone loss, using data from the KNHANES database. Frequent milk consumption was associated with a significantly high BMD and low OR for osteoporosis.

Previous studies on milk consumption and BMD have based their data on milk intake history during childhood or adolescence, and many are limited to the female population alone. ${ }^{8,9)}$ Sandler et al. ${ }^{13)}$ reported the effect of frequent milk consumption in childhood and adolescence on higher bone density during menopause. Similarly, Murphy et al. ${ }^{14)}$ found that frequent milk consumption before the age of 25

Table 2. Adjusted means (95\% confidence intervals) of BMDs in Korean adults (men) grouped by milk consumption frequency according to the Korea National Health and Nutrition Examination Survey 2008-2011 after covariates* adjustment

\begin{tabular}{|c|c|c|c|c|c|}
\hline \multirow{2}{*}{$\begin{array}{l}\text { Measurement } \\
\text { site }\end{array}$} & \multirow{2}{*}{$\begin{array}{l}\text { Frequency of milk } \\
\text { consumption }\end{array}$} & \multicolumn{4}{|c|}{$\mathrm{BMD}\left(\mathrm{g} / \mathrm{cm}^{2}\right)$} \\
\hline & & $<50$ years old $(n=2,969)$ & P-value & $\geq 50$ years old $(n=2,912)$ & P-value \\
\hline \multirow[t]{3}{*}{ Total femur } & $\leq 1 /$ wk & $0.994(0.987-1.002)$ & & $0.939(0.933-0.945)$ & \\
\hline & $2-6 / w k$ & $1.003(0.994-1.012)$ & 0.133 & $0.942(0.930-0.954)$ & 0.649 \\
\hline & $\geq 1 / d$ & $1.011(1.001-1.021)$ & 0.010 & $0.947(0.932-0.962)$ & 0.283 \\
\hline \multirow[t]{3}{*}{ Femur neck } & $\leq 1 /$ wk & $0.857(0.849-0.864)$ & & $0.760(0.754-0.765)$ & \\
\hline & $2-6 / w k$ & $0.865(0.856-0.874)$ & 0.144 & $0.762(0.751-0.774)$ & 0.702 \\
\hline & $\geq 1 / d$ & $0.875(0.865-0.886)$ & 0.005 & $0.768(0.753-0.782)$ & 0.294 \\
\hline \multirow[t]{3}{*}{ Lumbar spine } & $\leq 1 /$ wk & $0.986(0.979-0.993)$ & & $0.947(0.940-0.954)$ & \\
\hline & $2-6 / w k$ & $0.986(0.977-0.996)$ & 0.969 & $0.951(0.937-0.965)$ & 0.568 \\
\hline & $\geq 1 / d$ & $0.988(0.977-0.998)$ & 0.847 & $0.972(0.952-0.992)$ & 0.017 \\
\hline
\end{tabular}

Values are presented as mean ( $95 \%$ confidence interval). Bold letters emphasize the significant difference from reference ( $\leq 1 /$ wk) adjusted mean statistically. $\mathrm{BMD}$, bone mineral density.

${ }^{*}$ Age, body mass index, physical activity, serum 25-OH-vitamin D levels, daily calcium intake, frequency of alcohol intake, smoking, and diabetes.

Table 3. Adjusted means (95\% confidence intervals) of BMDs in Korean adults (women) grouped by milk consumption frequency according to the Korean National Health and Nutrition Examination Survey 2008-2011 after covariates ${ }^{\dagger}$ adjustment

\begin{tabular}{|c|c|c|c|c|c|}
\hline \multirow{2}{*}{$\begin{array}{l}\text { Measurement } \\
\quad \text { site }\end{array}$} & \multirow{2}{*}{$\begin{array}{l}\text { Frequency of milk } \\
\text { consumption }\end{array}$} & \multicolumn{4}{|c|}{$\mathrm{BMD}\left(\mathrm{g} / \mathrm{cm}^{2}\right)$} \\
\hline & & Pre-menopause $(n=1,735)$ & P-value & Post-menopause $(n=1,233)$ & P-value \\
\hline \multirow[t]{3}{*}{ Total femur } & $\leq 1 / w k$ & $0.894(0.885-0.903)$ & & $0.791(0.781-0.801)$ & \\
\hline & $2-6 /$ wk & $0.886(0.877-0.895)$ & 0.245 & $0.812(0.798-0.825)$ & 0.013 \\
\hline & $\geq 1 / d$ & $0.894(0.884-0.904)$ & 0.997 & $0.812(0.794-0.830)$ & 0.056 \\
\hline \multirow[t]{3}{*}{ Femur neck } & $\leq 1 /$ wk & $0.766(0.756-0.775)$ & & $0.650(0.642-0.659)$ & \\
\hline & $2-6 /$ wk & $0.765(0.755-0.774)$ & 0.890 & $0.659(0.645-0.672)$ & 0.312 \\
\hline & $\geq 1 / d$ & $0.767(0.754-0.779)$ & 0.921 & $0.664(0.646-0.683)$ & 0.207 \\
\hline \multirow[t]{3}{*}{ Lumbar spine } & $\leq 1 /$ wk & $0.987(0.978-0.997)$ & & $0.835(0.822-0.847)$ & \\
\hline & $2-6 /$ wk & $0.982(0.970-0.994)$ & 0.470 & $0.844(0.829-0.859)$ & 0.299 \\
\hline & $\geq 1 / d$ & $0.980(0.967-0.993)$ & 0.375 & $0.851(0.825-0.877)$ & 0.257 \\
\hline
\end{tabular}

Values are presented as mean ( $95 \%$ confidence interval). Bold letters emphasize the significant difference from reference ( $\leq 1 /$ wk) adjusted mean statistically. $\mathrm{BMD}$, bone mineral density.

*Age, body mass index, physical activity, serum 25-OH-vitamin D levels, daily calcium intake, frequency of alcohol intake, smoking, diabetes, and hormonal therapy for women. 
Table 4. ORs $(95 \% \mathrm{Cls})^{\star}$ for osteoporosis (T score $\left.\leq-2.5\right)$ in men aged 50 or above and postmenopausal women according to milk consumption frequency after covariates ${ }^{\dagger}$ adjustment

\begin{tabular}{|c|c|c|c|}
\hline Measurement site & Frequency of milk consumption & OR $(95 \% \mathrm{Cl})$ & P-value \\
\hline \multicolumn{4}{|l|}{ Men $(n=2,912)$} \\
\hline \multirow[t]{3}{*}{ Total femur } & $\leq 1 /$ wk & 1 & \\
\hline & $2-6 /$ wk & $0.31(0.062-1.550)$ & 0.153 \\
\hline & $\geq 1 / d$ & $0.29(0.030-2.815)$ & 0.284 \\
\hline \multirow[t]{3}{*}{ Femur neck } & $\leq 1 /$ wk & 1 & \\
\hline & $2-6 / w k$ & $0.15(0.055-0.409)$ & 0.000 \\
\hline & $\geq 1 / d$ & $0.35(0.125-0.979)$ & 0.046 \\
\hline \multirow[t]{3}{*}{ Lumbar spine } & $\leq 1 /$ Wk & 1 & \\
\hline & $2-6 /$ wk & $0.84(0.474-1.494)$ & 0.555 \\
\hline & $\geq 1 / d$ & $0.34(0.143-0.804)$ & 0.014 \\
\hline \multicolumn{4}{|l|}{ Women $(n=1,233)$} \\
\hline \multirow[t]{3}{*}{ Total femur } & $\leq 1 /$ wk & 1 & \\
\hline & $2-6 / w k$ & $0.23(0.055-0.958)$ & 0.044 \\
\hline & $\geq 1 / d$ & $0.55(0.117-2.610)$ & 0.452 \\
\hline \multirow[t]{3}{*}{ Femur neck } & $\leq 1 /$ wk & 1 & \\
\hline & $2-6 / w k$ & $0.61(0.352-1.071)$ & 0.085 \\
\hline & $\geq 1 / d$ & $0.47(0.203-1.096)$ & 0.080 \\
\hline \multirow[t]{3}{*}{ Lumbar spine } & $\leq 1 /$ wk & 1 & \\
\hline & $2-6 /$ wk & $0.69(0.441-1.086)$ & 0.109 \\
\hline & $\geq 1 / d$ & $0.65(0.339-1.245)$ & 0.193 \\
\hline
\end{tabular}

Bold letters emphasize statistically significant odds ratio difference $(\mathrm{P}<0.01, \mathrm{P}<0.05)$.

$\mathrm{OR}$, odds ratio; $\mathrm{Cl}$, confidence interval.

${ }^{*}$ ORs and $95 \%$ Cls were obtained using binary logistic regression. ${ }^{\dagger}$ Confounding variables include age, body mass index, physical activity, serum $25-0 \mathrm{H}$-vitamin $\mathrm{D}$ levels, daily calcium intake, frequency of alcohol intake, smoking, diabetes, and hormonal therapy for women.

years influenced hip bone mass in middle-aged and older women in Cambridge, United Kingdom. A study on both elderly women and men (aged 66-96 years) in Iceland revealed a strong association between midlife milk consumption and BMD in old age. In this study, a food frequency questionnaire was used to obtain data pertaining to the frequency of milk consumption during adolescence, midlife (age 40-50 years), and the present moment; however, BMD was measured using quantitative computed tomography, and the study did not include young adults. ${ }^{15)}$ Additionally, the populations assessed in these reports have usually been females and belonged to particular age groups.

A few studies in Korea have shown an association between milk consumption, BMD, and osteoporosis. In a 4-year prospective cohort study on postmenopausal women, a milk intake exceeding 5-6 servings per week decreased the risk of radial but not tibial osteoporosis (hazard ratio, 0.60; 95\% CI, 0.42-0.87) compared with 0 weekly servings. ${ }^{16)}$ Studies have also been conducted using the KNHANES. Hong et al. ${ }^{17)}$ found varying degrees of osteoporosis risk compared to those who consumed no dairy; those who consumed either less than once or more than once daily had an OR of either 0.96 (95\% CI, $0.66-1.38$ ) or 0.71 (95\% CI, 0.53-0.96), respectively. Although this study included adults aged over 20 years, the consumption of milk was not discriminated from that of dairy products. Similarly, Baek et al. ${ }^{18)}$ used the KNHANES and found that men (aged 30-39 years) who consumed milk more than once per day had a higher femur BMD, a finding that reflects those of or study. The OR for osteoporosis was lower in the group that consumed milk more than once per day, but specific bone sites were not analyzed. In addition, bone loss has been found to occur at an older age in men than in women. However, the population of the study was between 19 and 64 years of age, a range that does not take into consideration elderly men.

Several mechanisms explain the association between the frequency of milk consumption and BMD. First, the direct and indirect effects of various components of milk may collectively promote increased BMD. Milk is composed of inorganic ions, such as calcium, magnesium, potassium, lactose, vitamins, fats, and proteins. In addition, lactose ${ }^{19)}$ and casein ${ }^{20)}$ are known to enhance calcium absorption, while milk protein stimulates insulin-like growth factor I and promotes bone formation. ${ }^{21)}$ Second, milk consumption may influence BMD by means of skeletal muscle. Milk consumption frequency has been suggested to be associated with skeletal muscle mass and strength, which are known to correlate with BMD and osteoporosis in both young and elderly adult populations. ${ }^{22-24)}$ Finally, milk consumption may increase bone mineral content through the inhibition of bone resorption. In a meta-analysis, milk had a significant effect on bone metabolism and BMD. ${ }^{25)}$

Our results showed that frequent milk consumption was associated with a low OR for osteoporosis, particularly at the lumbar spine in men aged 50 years or above and in the total femur in postmenopausal women. A high BMD was observed in the same bone sites when milk was consumed frequently. This tendency based on sex may be due to the site-specific pattern of cortical and trabecular bone loss, as well as, men having a predisposition to greater periosteal apposition and a 
higher BMD in the hip during aging. ${ }^{26,27)}$ Thus, the effect of milk consumption may not be as significant at this site in men as it is in women. Consequently, in women, a low OR for osteoporosis was observed in the total femur. Both a continued habit of milk consumption during aging and the influence of milk consumption on BMD later in life may have positively influenced BMD and the OR for osteoporosis in adults aged 50 years or above. This explanation is supported by the findings of prior reports in postmenopausal women. ${ }^{13)}$

In contrast to premenopausal women, men aged $<50$ years who consumed milk more than once per day had high femoral BMD in our study. In a population-based study from the KNHANES IV (20082009), peak bone mass was achieved at age 21 years in men, and a subsequent accelerated phase of age-related bone loss was observed in the femur. ${ }^{28)}$ Specifically, the mean annual rate of bone loss in the femurs of men aged 23-29 years was highest among those aged 10-80 years in both sexes. In addition, BMD at the lumbar spine increased until the age of 28 years in women. Another study by Kristensen et al. ${ }^{29)}$ showed that 10 days of milk intake significantly decreased bone turnover in young men aged 22-29 years. Thus, femoral BMD and BMD in men aged $<50$ years may be more susceptible to change by modifiable factors, such as an increased frequency of milk intake. Factors other than milk consumption, such as estrogen levels, may play a greater role in BMD during early adulthood in women. ${ }^{30)}$

This study has several limitations. First, as milk is composed of several nutrients, the association observed between milk consumption and BMD cannot be explained by one particular component. Although the positive effect of milk on BMD is often attributed to calcium, other milk components may also have an effect. In our study, the results were not significantly affected by adjusting for daily calcium intake. These findings suggest that the positive effect of milk on BMD may not be solely due to calcium intake. Second, milk intake was measured by frequency rather than by the total amount, and the type of milk was not specified (for example, low-fat milk). In addition, the dietary questionnaire may not accurately represent an individual's daily diet, and any supplements or other dairy products may not have been accounted for. Third, data used in this study were limited to the KNHANES conducted from 2008 to 2011. Subsequent surveys did not include BMD and therefore, could not be used for this study. Fourth, medications that may affect BMD, such as steroid and hormone preparations, could not be adjusted for in the analysis. Finally, this crosssectional study does not provide an explanation for the cause-and-effect relationship between the frequency of milk consumption and BMD. Despite these limitations, the results of our study are generalizable to the adult population as a whole and are not limited to a certain age group or sex. The use of standardized DXA measurements and adjustments for multiple covariates enhance the reliability of our findings.

In conclusion, frequent milk consumption was associated with a significantly high BMD and reduced OR for osteoporosis. In postmenopausal women, frequent milk consumption was associated with a high BMD and low OR for osteoporosis in the total femur; men aged
50 years or above showed similar results in the lumbar spine alone, and moreover, a low OR for osteoporosis at the femur neck. Furthermore, in men aged $<50$ years, consumption of milk more than once per day was associated with a high femoral BMD. Further prospective study is necessary to elucidate the effect of milk consumption on BMD.

\section{CONFLICT OF INTEREST}

No potential conflict of interest relevant to this article was reported.

\section{ORCID}

Ji Soo Kim : https://orcid.org/0000-0002-1363-0840

Seung-Won Oh: https://orcid.org/0000-0003-3800-0754

Jiwoo Kim: https://orcid.org/0000-0002-4582-9184

\section{REFERENCES}

1. Choi YJ, Oh HJ, Kim DJ, Lee Y, Chung YS. The prevalence of osteoporosis in Korean adults aged 50 years or older and the higher diagnosis rates in women who were beneficiaries of a national screening program: the Korea National Health and Nutrition Examination Survey 2008-2009. J Bone Miner Res 2012;27:1879-86.

2. Cumming RG, Nevitt MC. Calcium for prevention of osteoporotic fractures in postmenopausal women. J Bone Miner Res 1997;12:1321-9.

3. Dawson-Hughes B. Osteoporosis treatment and the calcium requirement. Am J Clin Nutr 1998;67:5-6.

4. Holbrook TL, Barrett-Connor E, Wingard DL. Dietary calcium and risk of hip fracture: 14-year prospective population study. Lancet 1988;2: 1046-9.

5. Ministry of Health and Welfare; The Korean Nutrition Society. Dietary reference intakes for Koreans 2015. Seoul: The Korean Nutrition Society; 2015.

6. Park J, Lee HS, Lee C, Lee HJ. Milk consumption patterns and perceptions in Korean adolescents, adults, and the elderly. Int Dairy J 2019; 95:78-85.

7. Ministry of Health and Welfare; Korea Centers for Disease Control and Prevention. Korea health statistics 2018: Korea National Health and Nutrition Examination Survey (KNHANES VII-3). Sejong: Ministry of Health and Welfare; 2018.

8. Teegarden D, Lyle RM, Proulx WR, Johnston CC, Weaver CM. Previous milk consumption is associated with greater bone density in young women. Am J Clin Nutr 1999;69:1014-7.

9. Kalkwarf HJ, Khoury JC, Lanphear BP. Milk intake during childhood and adolescence, adult bone density, and osteoporotic fractures in US women. Am J Clin Nutr 2003;77:257-65.

10. Du XQ, Greenfield H, Fraser DR, Ge KY, Liu ZH, He W. Milk consumption and bone mineral content in Chinese adolescent girls. Bone 2002;30:521-8.

11. Cho DS, Lee JY. Bone mineral density and factors affecting in female college students. Korean J Women Health Nurs 2008;14:297-305.

12. Mun SO, Kim J, Yang YJ. Factors associated with bone mineral density 
in Korean postmenopausal women aged 50 years and above: using 2008-2010 Korean National Health and Nutrition Examination Survey. Korean J Community Nutr 2013;18:177-86.

13. Sandler RB, Slemenda CW, LaPorte RE, Cauley JA, Schramm MM, Barresi ML, et al. Postmenopausal bone density and milk consumption in childhood and adolescence. Am J Clin Nutr 1985;42:270-4.

14. Murphy S, Khaw KT, May H, Compston JE. Milk consumption and bone mineral density in middle aged and elderly women. BMJ 1994; 308:939-41.

15. Eysteinsdottir T, Halldorsson TI, Thorsdottir I, Sigurdsson G, Sigurdsson S, Harris T, et al. Milk consumption throughout life and bone mineral content and density in elderly men and women. Osteoporos Int 2014;25:663-72.

16. Park SJ, Jung JH, Kim MS, Lee HJ. High dairy products intake reduces osteoporosis risk in Korean postmenopausal women: a 4 year followup study. Nutr Res Pract 2018;12:436-42.

17. Hong H, Kim EK, Lee JS. Effects of calcium intake, milk and dairy product intake, and blood vitamin D level on osteoporosis risk in Korean adults: analysis of the 2008 and 2009 Korea National Health and Nutrition Examination Survey. Nutr Res Pract 2013;7:409-17.

18. Baek SW, Lee HO, Kim HJ, Won ES, Ha YS, Shin YK, et al. Relationship between intake of milk and milk products and bone health by sex and age-group in Koreans: using data from the Korea National Health and Nutrition Examination Survey 2008-2011. J Korean Soc Food Sci Nutr 2017;46:513-22.

19. Kobayashi A, Kawai S, Obe Y, Nagashima Y. Effects of dietary lactose and lactase preparation on the intestinal absorption of calcium and magnesium in normal infants. Am J Clin Nutr 1975;28:681-3.

20. Sato R, Noguchi T, Naito H. Casein phosphopeptide (CPP) enhances calcium absorption from the ligated segment of rat small intestine. J Nutr Sci Vitaminol (Tokyo) 1986;32:67-76.
21. Rizzoli R. Dairy products, yogurts, and bone health. Am J Clin Nutr 2014;99(5 Suppl):1256S-1262S.

22. Lee JH, Lee HS, Kim H, Kwon YJ, Lee JW. Association of milk consumption frequency on muscle mass and strength: an analysis of three representative Korean population studies. Eur J Nutr 2020;59:3257-67.

23. Bierhals IO, Dos Santos Vaz J, Bielemann RM, de Mola CL, Barros FC, Goncalves $\mathrm{H}$, et al. Associations between body mass index, body composition and bone density in young adults: findings from a Southern Brazilian cohort. BMC Musculoskelet Disord 2019;20:322.

24. Kim S, Won CW, Kim BS, Choi HR, Moon MY. The association between the low muscle mass and osteoporosis in elderly Korean people. J Korean Med Sci 2014;29:995-1000.

25. Ma DF, Zheng W, Ding M, Zhang YM, Wang PY. Milk intake increases bone mineral content through inhibiting bone resorption: meta-analysis of randomized controlled trials. e-SPEN J 2013;8:e1-7.

26. Eckstein F, Matsuura M, Kuhn V, Priemel M, Muller R, Link TM, et al. Sex differences of human trabecular bone microstructure in aging are site-dependent. J Bone Miner Res 2007;22:817-24.

27. Alswat KA. Gender disparities in osteoporosis. J Clin Med Res 2017;9: 382-7.

28. Lee EY, Kim D, Kim KM, Kim KJ, Choi HS, Rhee Y, et al. Age-related bone mineral density patterns in Koreans (KNHANES IV). J Clin Endocrinol Metab 2012;97:3310-8.

29. Kristensen M, Jensen M, Kudsk J, Henriksen M, Molgaard C. Shortterm effects on bone turnover of replacing milk with cola beverages: a 10-day interventional study in young men. Osteoporos Int 2005;16: $1803-8$.

30. Ho-Pham LT, Nguyen ND, Nguyen TV. Quantification of the relative contribution of estrogen to bone mineral density in men and women. BMC Musculoskelet Disord 2013;14:366. 